\title{
Paul Decauville et le tourisme ferroviaire : un « modèle Decauville »
}

Paul Decauville and railway tourism: the "Decauville model "

Jean-Jacques Marchi

\section{OpenEdition}

\section{Journals}

Édition électronique

URL : https://journals.openedition.org/rhcf/1569

DOI : 10.4000/rhcf.1569

Éditeur

Rails \& histoire

Édition imprimée

Date de publication : 1 septembre 2012

Pagination : 233-251

ISSN : 0996-9403

\section{Référence électronique}

Jean-Jacques Marchi, «Paul Decauville et le tourisme ferroviaire : un « modèle Decauville » », Revue

d'histoire des chemins de fer [En ligne], 42-43 | 2012, mis en ligne le 14 novembre 2014, consulté le 22 avril 2022. URL : http://journals.openedition.org/rhcf/1569; DOI : https://doi.org/10.4000/rhcf.1569 
Jean-Jacques MARCHI

\section{Paul Decauville et le tourisme ferroviaire : un « modèle Decauville "}

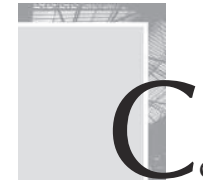

t article, issu des recherches de l'auteur ${ }^{1}$, vise à préciser la contribution peu connue de l'inventeur et entrepreneur Paul Decauville au développement du tourisme ferroviaire ${ }^{2}$. En effet, les productions des Établissements Decauville participent à l'essor de formes ludiques du tourisme ferroviaire dès la fin des années 1870 . Près d'un siècle plus tard, les matériels ayant survécu ainsi que des productions plus récentes sont réutilisés au profit de formes de tourisme ferroviaires fondées sur la mise en valeur du patrimoine.

1- MARCHI, 2007, 2010.

2- Nous entendons par tourisme ferroviaire des pratiques de tourisme ou de loisirs effectuées par chemin de fer, à un rythme lent, pour l'attrait que présente le mode ferroviaire en luimême : découverte de paysages, contact avec un patrimoine ferroviaire «vivant », vécu d'expériences originales. Dans ce cadre, le besoin éventuel de transport ne constitue pas la motivation principale. Si le terme de tourisme ferroviaire est relativement récent (il daterait des années 1950-1960), les pratiques observées dès les années 1870 en relèvent visiblement. 


\section{Les chemins de fer ludiques : des petits trains anciens et méconnus}

«À l'origine, le tourisme ferroviaire s’inspire d'un intérêt renouvelé pour le paysage. Cette inspiration est typiquement romantique et elle se situe dans le cadre d'un retour à la nature qui débouchait, en particulier, sur une nouvelle attitude favorable aux beautés des Alpes [...] le but du voyage n'est pas seulement de rejoindre un endroit déterminé, mais le déplacement lui-même acquiert une fascination qu'il n'avait pas eue auparavant. Nous assistons à la naissance du voyage panoramique, c'est-à-dire du voyage qui a déjà une valeur en lui-même ${ }^{3}$."

Cet attrait pour le voyage panoramique ne se limite pas aux montagnes : les espaces littoraux, considérés autrefois comme répulsifs, sont l'objet à la fin du XIX ${ }^{\mathrm{e}}$ siècle d'un intérêt nouveau ${ }^{4}$. Tandis que les trains à crémaillère montent à l'assaut des cimes, un nouveau genre de chemin de fer apparaît sur les rivages maritimes : des petits trains à caractère ludique qui ont pour principal objectif de promener et/ou divertir leurs visiteurs (ils peuvent également assurer une fonction de déplacement au cour d'une zone d'animation). Ces petits trains éclosent aussi dans des lieux plus familiers : les parcs et jardins publics dont la fréquentation est liée aux pratiques thermales ou de ce que nous appelerions aujourd'hui les loisirs de proximité (la sortie du dimanche).

Les petits trains ludiques sont peu présents dans les écrits académiques. Ils sont aussi parfois 'snobés' par les milieux 'ferrovipathes' contemporains. Est-ce du fait de leur caractère futile, amusement et promenade n'étant pas considérés, en eux-mêmes, comme des occupations sérieuses ? Ou la conséquence d'existences éphémères ou erratiques ? Pourtant, contemporains des trains à crémaillère ${ }^{5}$, les petits trains ludiques participent comme eux à l'essor du tourisme et des loisirs.

À la Pointe-de-Grave (Gironde), le 'réseau des Huttes', à voie métrique, doit approvisionner en matériaux le chantier destiné à protéger la côte des attaques de l'océan. Il propose, dès le Second Empire, des balades aux excursionnistes et touristes venant de Soulac ou de Royan : "L'administration des Ponts et Chaussées livre avec courtoisie ses wagons et son chemin de fer américain qui permet de visiter la majestueuse jetée de la Pointe-de-Grave, le phare du

3- GIUNTINI, 2003, p. 60-61. Il s'agit bien d'une nouvelle attitude : la montagne inspirait auparavant de la répulsion. Remarquons que, avant les Romantiques, les écrits de Jean-Jacques Rousseau (1712-1778) ont contribué à renouveler la perception des paysages montagnards.

4- Alain Corbin (CORBIN, 1988) parle de « désir de rivage ».

5- 1869 voit la mise en service du premier train à crémaillère, le « Railway to the Moon » ou chemin de fer du Mont Washington (New Hampshire, États-Unis). L'année suivante est inauguré le train à crémaillère du Righi (Suisse), premier train à crémaillère d'Europe. 
Grave, les vingt-sept épis protecteurs de la côte, la délicieuse villa dite maison de Grave [...], le sémaphore ou tour de Saint-Nicolas [...], l'anse des Huttes avec ses admirables travaux ${ }^{6}$. " Ainsi, " ce service insolite peut sans doute être considéré comme un ancêtre des chemins de fer touristiques ${ }^{7}$.

Autre exemple de réseau à double fonction, le tramway forestier de la Coubre : établi vers 1870 par le service des Eaux et Forêts pour boiser les dunes et limiter l'érosion marine, il est concédé à la fois pour l'exploitation forestière et les promenades touristiques qu'affectionnent les touristes en villégiature à Royan. En 1878 est inauguré à Paris, porte Maillot, le petit train du Jardin d'Acclimatation ${ }^{8}$. Sur la presqu'île du Cap Ferret (Gironde), différents tramways roulent bien avant celui que l'on peut emprunter encore aujourd'hui : le premier d'entre eux est établi en 1879.

Comme pour les trains à crémaillère, certaines innovations techniques et commerciales ont permis un large développement des petits trains ludiques. L'inventeur et entrepreneur Paul Decauville a, dans ce cadre, joué un rôle que nous nous proposons d'étudier.

\section{Paul Decauville, les voies du succès ${ }^{9}$}

\section{Du « porteur " aux passagers}

Paul Decauville (1846-1922) est l'inventeur de la voie ferrée qui porte son nom. Il s'agit d'une voie ferrée étroite ( 40,50 ou $60 \mathrm{~cm}$ d'écartement) dont la conception permet la pose et dépose faciles. La voie est constituée de sections complètes (rails et traverses) qu'il suffit d'assembler les unes avec les autres, à la manière d'un jeu de construction, pour former des lignes voire des réseaux complets. On parle ainsi du 'porteur Decauville'.

6- CHANUC et DURBAIN, 2005, p. 214, citant L'Écho de Soulac de 1867. Le terme de « chemin de fer américain » mériterait des recherches complémentaires. Il est généralement utilisé comme synonyme de tramway. S'agit-il d'une référence aux pionniers américains, construisant à l'époque des chemins de fer légers pour mettre en valeur leur immense territoire?

7- Ibidem.

8- Nous reviendrons sur le tramway forestier de la Coubre et sur le petit train du Jardin d'Acclimatation.

9- Les informations figurant dans cette section sont issues de l'ouvrage, très documenté, de Roger Bailly (BAILLY, 1989), dans une moindre mesure de ACOVA, 2002, encart p. 9 et de DOLLFUS et GEOFFROY, 1938. 
Paul Decauville est issu d'une famille d'agriculteurs aisés devenus peu à peu de véritables industriels. Lorsque Paul arrive aux affaires ${ }^{10}$, les ateliers Decauville réalisent déjà des ouvrages importants destinés aux grandes compagnies de chemins de fer (châteaux d'eaux, ponts métalliques, chaudières, réchauffeurs, etc.). Les compétences acquises par la société en construction mécanique et en chaudronnerie constitueront des atouts décisifs dans la mise au point rapide du 'porteur' ${ }^{\prime 11}$.

Le vrai tournant dans la vie professionnelle de Paul Decauville est pris en 1875, année durant laquelle il est confronté à un défi : une récolte de betteraves exceptionnelle ( 9000 tonnes). Il a beaucoup plu et le système traditionnel de transport par tombereau s'avère inutilisable. Refusant de perdre la récolte, Paul Decauville voit dans ces circonstances inhabituelles l'occasion de tester en vraie grandeur son chemin de fer portatif que Roger Bailly nous décrit ainsi : des "éléments portatifs constitués à l'origine par deux fers carrés parallèles fixés sur des traverses de fer plat et écartés de quarante centimètres, analogues à des échelles et pesant chacun une quarantaine de kilogrammes, ce qui permettait leur déplacement avec facilité par un seul homme, existaient déjà à l'automne 1875. Sur ces 'rails' rudimentaires roulaient de petits wagonnets à deux essieux, sur lesquels on pouvait, en se jouant de la boue, transporter des paniers contenant une centaine de kilogrammes de betteraves [...]. Au tombereau et à la brouette, Paul Decauville ajoutait un nouveau moyen de manutention : le wagonnet !... Cette invention qui survenait à un moment où elle répondait à un besoin urgent de la plupart des activités industrielles allait connaître un succès extraordinaire ${ }^{12}$."

Le test est concluant, la récolte est sauvée. Le système est perfectionné et son emploi généralisé au sein de l'exploitation agricole : transport des pièces, évacuation du fumier, etc. Le catalogue Decauville vante bientôt un large éventail d'utilisations (du maraîchage au réseau militaire en passant par les mines et les carrières) et propose d'autres écartements de voie mieux adaptés à des charges plus lourdes $(50 \mathrm{~cm}$ et surtout $60 \mathrm{~cm}$ qui deviendra par la suite la référence).

10- Depuis 1864, Paul Decauville aide son père qui souffre de problèmes de santé. En 1871, au décès de ce dernier, il se retrouve seul maittre à bord.

11- BAILLY, 1989, p. 12.

12- Ibidem, p. 15. Contrairement à ce que l'on peut lire souvent, Roger Bailly affirme que le petit chemin de fer n'a pas été inventé à l'occasion de cette récolte extraordinaire puisqu'il figurait déjà au catalogue de la maison Decauville, paru quelques mois auparavant. 
La possibilité d'appliquer son système au transport de passagers se révèle à Paul Decauville à l'occasion d'un voyage sur un chemin de fer gallois qui constitue la vitrine de l'industrie britannique pour la voie étroite, le Ffestiniog Railway ${ }^{13}$. «Paul Decauville voyait l'avenir de son Entreprise, bien au-delà de la production du simple 'porteur' puisque déjà en 1876, ayant entendu parler du chemin de fer à l'écartement de 0,61 m (deux pieds anglais), qui, au Pays de Galles, de Festiniog à Pormadoc, fonctionnait depuis 1832, pour la desserte des ardoisières, sur une longueur de $23 \mathrm{~km}$, se rendit en Angleterre afin de faire connaissance avec ce petit réseau [...]. À l'origine, là aussi, la traction avait été assurée par des chevaux mais depuis 1864, neuf locomotives avaient pris le relais [...]. Ce chemin de fer transportait des voyageurs et des marchandises [...]. Paul Decauville fut conquis et, tirant les conséquences de sa visite, il se proposa de développer en France ce système ${ }^{14}$. "

\section{Des lignes de démonstration et de promenades publiques}

Aussitôt rentré chez lui, Paul Decauville porte à $50 \mathrm{~cm}$ puis à $60 \mathrm{~cm}$ l'écartement de la ligne à voie métrique ${ }^{15}$ desservant ses carrières, essaie dans sa propriété une locomotive ainsi que du matériel roulant destiné au transport de marchandises et de passagers. En 1877, la ligne provisoire installée à l'occasion $\mathrm{du}$ Concours de Compiègne constitue la première application en France de la voie étroite au transport de passagers. Lilliput, locomotive à vapeur à voie de $50 \mathrm{~cm}$, y promène 60 personnes à la fois d'un bout à l'autre du concours ${ }^{16}$. Doté d'un sens de la communication aiguisé, Paul Decauville multiplie les lignes de démonstration et de promenades publiques qui joueront un rôle important dans la diffusion des matériels ferroviaires à voie étroite. Elles constitueront les vitrines du savoir-faire des Établissements Decauville ${ }^{17}$.

13- Voir MARCHI, 2010, p. 339-340.

14- BAILLLY, 1989, p. 18. Un extrait du catalogue Decauville de 1884 indique que Paul Decauville a fait trois voyages au Pays-de-Galles « pour étudier toutes les lignes à voie étroite de $0,60 \mathrm{~m}$ qui y fonctionnent depuis cinquante ans et dont la plupart transportent les voyageurs ». Ibidem, ill. p. 155.

15- La voie métrique semble constituer jusque-là un standard pour les réseaux 'légers' ('réseau des Huttes', tramway de la Coubre).

16- Selon le catalogue Decauville de 1887, Lilliput est ensuite envoyée à Rotterdam, où elle roule «sur un chemin de fer circulaire [...] pour l'amusement des Hollandais!». Car « une locomotive aussi petite ne pouvait faire un service sérieux ", ibid., p. 99.

17- Entre 1877 et 1890, MARCHI, 2010, p. 574, en recense dix-sept, sur la base des informations fournies par BAILLY, 1989. 


\section{Le petit train du Jardin d'Acclimatation}

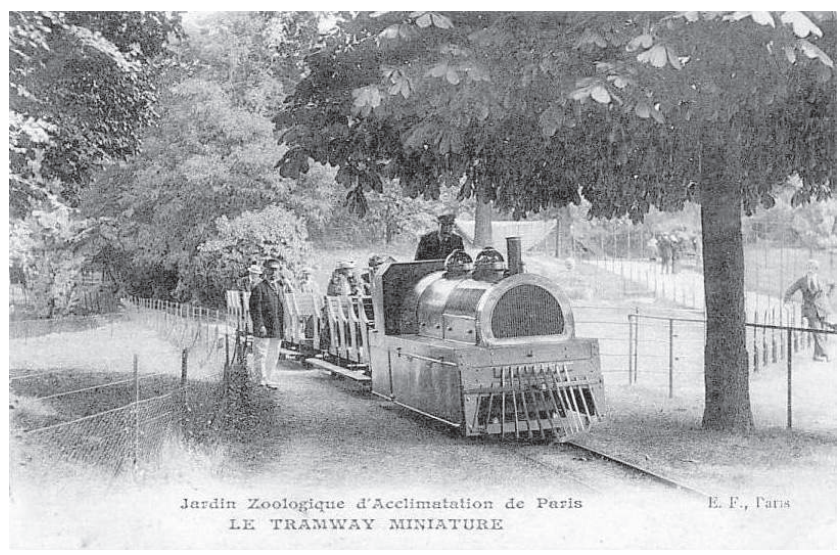

Figure 1. Jardin zoologique d'Acclimatation de Paris - Le tramway miniature. Carte postale, vers 1920 . Noter la fausse locomotive à vapeur.

En 1878, la voie de Decauville de $50 \mathrm{~cm}$ est choisie pour l'acheminement des colis des exposants de l'Exposition Universelle. À l'ouverture de l'exposition, Paul Decauville a l'idée d'utiliser la ligne pour le transport des visiteurs. Il propose au responsable de l'exposition "d'installer, à ses risques et périls, un petit tramway entre le Trocadéro et l'École Militaire ${ }^{18}$, mais celuici refuse. Paul Decauville se tourne alors vers le Jardin d'Acclimatation du Bois de Boulogne qui accepte son petit train.

Première application de la voie étroite au transport régulier de passagers, le petit train du Jardin d'Acclimatation se présente sous la forme de wagonnets découverts dans lesquels les voyageurs s'installent sur des banquettes placées dos à dos (fig. 1). Chaque train est tiré par deux poneys ${ }^{19}$, à la vitesse de $15 \mathrm{~km} / \mathrm{h}$ environ. La ligne s'étend sur 1500 mètres. Dans des conditions de sécurité parfaites (" aucun déraillement ne s'est jamais produit $»^{20}$ ), elle transporte un nombre considérable de voyageurs : en moyenne 10000 à 15000 voyageurs par mois, avec des pointes à 3000 certains dimanches après-midi ${ }^{21}$. Ainsi, en 1878, incontestablement, le chemin de fer touristique à caractère ludique est né2 ${ }^{2}$. Le succès du petit train du Jardin d'Acclimatation

18- BAILLY, 1989, p. 16.

19- Les poneys seront remplacés par des locotracteurs vers 1910. Voir aussi note 36.

20- Ibidem.

21- Ibid. Une telle fréquentation en fait, à l'époque déjà, un phénomène de masse. Aujourd'hui encore, le petit train du Jardin d'Acclimatation est probablement le plus visité en France dans sa catégorie.

22- Nous abordons plus loin la question de savoir si les réalisations de ce type étaient effectivement perçues comme ludiques par leurs contemporains. 
suggère l'idée de promouvoir d'autres chemins de fer de ce type dans des stations balnéaires ${ }^{23}$. Il constitue par la suite une référence. Ainsi, dans le guide de Victor Billaud, Royan et ses environs, publié quelque dix ans plus tard, ne liton pas : « on trouve à l'entrée du parc un tramway semblable à celui du Jardin d'Acclimatation, qui dessert la forêt en suivant les grandes avenues intérieures et le boulevard de la mer $»^{24}$ ?

\section{La consécration, I'Exposition Universelle de 1889}

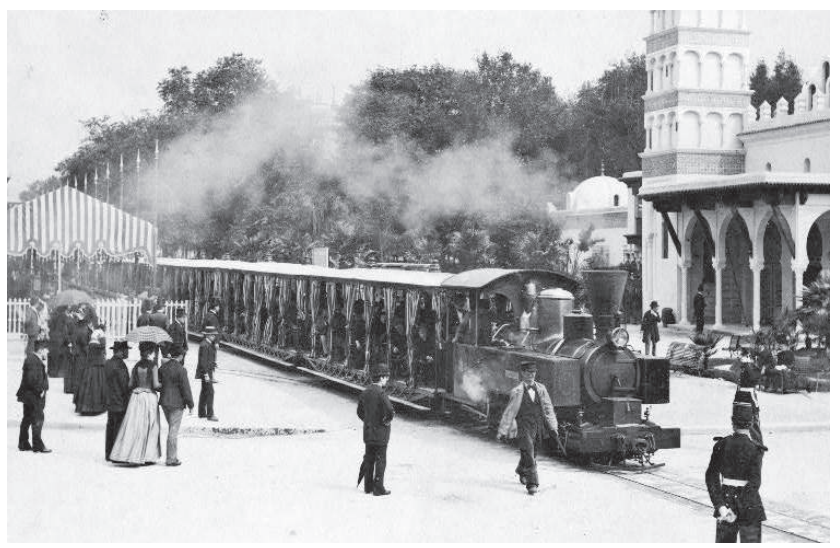

Figure 2. Le petit train à l'Exposition Universelle de 1889. Library of Congress, coll. Albert Tissandier, Prints \& Photographs Division [reproduction number : LC-USZ62-101100].

Mais, " ce qui fit la renommée du matériel du chemin de fer Decauville dans le monde entier, ce fut l'Exposition de 1889 au Champ-de-Mars ${ }^{25}$. Du palais des Machines à l'esplanade des Invalides, en passant devant la Tour Eiffel et en longeant le quai d'Orsay, il y avait trois kilomètres à parcourir. Le chemin de fer Decauville rendit ce service, et rien n'était plus pittoresque que de voir ces petits trains remplis d'une foule joyeuse. Du 6 mai au 6 novembre circulèrent 42500 trains, transportant 6342446 voyageurs, soit 35230 voyageurs par jour ${ }^{26}$. L'exploitation se déroula sans aucun accident, faisant la démonstration de la fiabilité des matériels Decauville et, aussi, de leur capacité à écouler, malgré leur format réduit (voie de $60 \mathrm{~cm}$ ), un flux massif de visiteurs (fig. 2).

La réaction des visiteurs révèle un engouement pour le 'Decauville'. Le journaliste de L'Abeille de Seine-et-Oise s'enthousiasme : «Continue donc, petit

23- «Une idée qui allait faire son chemin : 'De semblables applications peuvent être faites dans un certain nombre de villes de bains de mer' avait-on pu trouver à ce moment ». Ibid.

24- BILLAUD, 1890, p. 118.

25- Il s'agit de l'Exposition Universelle de 1889.

26- DOLLFUS et GEOFFROY, 1938, p. 244. 
chemin de fer, en amusant les voyageurs. Tu pourras prendre pour devise 'Utile dulci ${ }^{27}$." Un confrère témoigne dans la Revue de l'Exposition Universelle : "le petit chemin de fer Decauville est tout pareil à un jouet qui marche [...] Tout cela évoque une idée de frêle, de coquet, de joli, on voudrait posséder un chemin de fer de ce calibre aimable dans sa propriété de Meudon ou de Suresnes [...] personne ne peut croire que ce train mignon puisse écraser quiconque [...] les gens étant serrés quatre par quatre, ainsi que des poupées emballées, ne peuvent se donner des airs de voyageurs sérieux [...] Malgré le sérieux des chefs de gare, on sent bien qu'ils sont là pour rire [...] tunnel joujou, tunnel pour rire, tunnel qui redevient simple tonnelle ${ }^{28}$. » Pas de doute, il s'agissait bien là d'un petit train à caractère ludique, perçu comme tel.

Conscient de la performance du petit train, et toujours habile en matière de communication, Paul Decauville, quelques jours avant la fermeture de l'Exposition Universelle, fait placarder tout au long du parcours une affiche ainsi rédigée : "Le chemin de fer Decauville remercie les six millions de voyageurs qui lui ont accordé leur confiance ${ }^{29}$. " Devenu un véritable phénomène de $\operatorname{mode}^{30}$, le "Decauville " connaît un succès mondial, pour des usages des plus variés : travaux publics, agriculture, carrières, mines, voies militaires, réseaux ferroviaires pionniers ${ }^{31}$. L'entreprise fabrique bientôt des matériels à tous écartements (voie normale comprise) et utilisant tous types de motorisation (moteurs à vapeur, électriques, à combustion interne). Ce succès lui vaut d'être concurrencée (et copiée) tant en France qu'à l'étranger ${ }^{32}$. Ainsi, lorsque, en 1895, Paul Decauville lâche les rênes de la société, l'impulsion est bel et bien donnée ${ }^{33}$.

\section{La belle époque des « petits trains des plages »}

Les Établissements Decauville prennent une part active dans la multiplication des 'petits trains des plages', généralement à voie de $60 \mathrm{~cm}$, établis dans les stations balnéaires. Ainsi, "c’est au cours des années 18801885 que la vogue des bains de mer, qui avait pris son essor sous le Second

27- BAILLY, 1989, p. 73.

28- Ibidem, p. 74-75.

29- Ibid., p. 75.

30- Plusieurs chansonnettes ont été composées sur le « Decauville».

31- Le premier réseau du Maroc est établi en voie de $60 \mathrm{~cm}$.

32- Citons pour la France les établissements Jules Weitz à Lyon, Pétolat à Dijon, Popineau près de Paris. Ibid., p. 77 et note 1, p. 89.

33- Paul Decauville s'intéressera par la suite aux dirigeables, puis inventera un procédé génial de revêtement des berges des cours d'eau, la « cuirasse Decauville». Ibid., p. 66. 
Empire, devait connaître, en France, un grand développement, facilité par la construction de nouvelles lignes de chemin de fer. Les 'petits trains des plages' devaient commencer à faire parler d'eux et connaître en général un succès facile qui allait durer jusqu'au premier conflit mondial (fig. 3). La voie Decauville convenait parfaitement pour ces chemins de fer [...] des mois d'été et le matériel roulant fabriqué par l'usine de 'Petit-Bourg' était assez économique ${ }^{34}$.

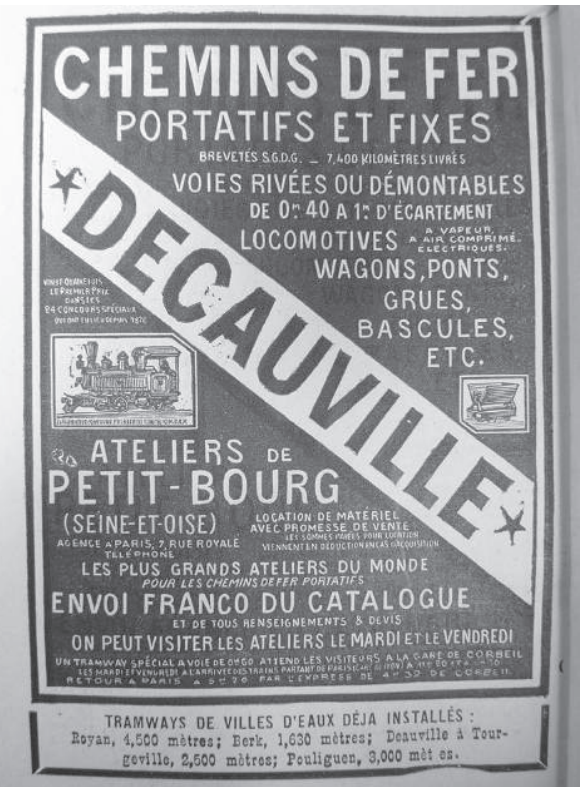
Toutefois les petits trains sont les victimes de la Grande Guerre et les survivants succombent à la montée en puissance du transport routier (autocar surtout) à partir des années 1920. Les Tramways de Royan constituent, par leur longévité, l'exception. Mais de quel(s) tramway(s) s'agit-il ?

Figure 3. Publicité Decauville parue dans Victor Billaud, Royan et ses environs, guide du touriste, Victor Billaud Editeur, Royan, 1890, p. Ixxxiv. Noter la référence aux " tramways de villes d'eaux déjà installés".

\section{Royan, quatre tramways, et quel rôle pour Decauville ${ }^{35}$ ?}

L'intérêt de la ville de Royan réside dans le fait qu'elle a connu plusieurs petits trains ludiques. Ils illustrent la diversité qui a existé au sein de cette catégorie et nous éclairent sur l'importance du rôle tenu par Decauville dans le développement du tourisme ferroviaire.

34- Ibid., p. 25. Petit-Bourg est le lieu d'implantation de l'usine historique des Établissements Decauville.

35- Les renseignements relatifs au tramway de la Coubre émanent de DEMONFAUCON, 2003, tandis que celles relatives aux Tramways de Royan proviennent essentiellement de BAILLY, 1989, p. 80 et notes correspondantes. Les interprétations qui en sont faites me sont évidemment personnelles. 


\section{Le tramway forestier de la Coubre}

Le premier petit train présent dans les environs de Royan est le tramway forestier de la Coubre, établi vers 1870 par l'administration des Eaux et Forêts dans la forêt du même nom. La zone est menacée par l'océan qui " grignote " progressivement les terres. Il faut donc boiser les dunes. En l'absence d'infrastructure, on choisit d'installer une voie ferrée pour acheminer le matériel nécessaire. La ligne, concédée à la fois pour l'exploitation forestière et pour les promenades touristiques, relie la Grande Côte et Ronce-les-Bains. La voie est à écartement métrique et armement léger. Les véhicules sont d'abord tirés par des chevaux. En 1903 l'exploitant met en service des automotrices à essence, la traction hippomobile subsistant en parallèle jusque dans les années $1930^{36}$.

Comme le 'réseau des Huttes', ce tramway est antérieur au système Decauville et sa conception est différente. Les premiers matériels n'ont rien à voir avec les voitures de style Decauville : les cartes postales anciennes montrent des sortes de carrioles sur rail ${ }^{37}$. Le choix de l'écartement des rails fait plutôt penser aux réseaux d'intérêt local amenés à se développer, surtout à partir des années 1880 suite à la loi de $1883^{38}$. Rien de 'Decauville' dans tout cela. Du moins, pas encore : en effet, en 1903, les automotrices à essence qui arrivent sur le réseau sont des fabrications des... Établissements Decauville.

\section{Les Tramways de Royan}

Les Tramways de Royan se caractérisent, dès le départ, par leur filiation directe avec Decauville. Le maire de Royan, M. Garnier, frappé par la démonstration éclatante du petit train lors de l'Exposition Universelle de 1889, voit dans ce moyen de transport l'opportunité de relier entre elles les plages de Royan qui s'étendent sur près de huit kilomètres. Il convainc les Établissements Decauville d'accepter la concession d'un tramway.

La cité balnéaire récupère le matériel de l'Exposition, devenu inutile, et dès 1890 ont lieu les premières circulations. Le tramway est à traction vapeur, avec des baladeuses des deux classes, et des voitures fermées. Écologiques avant

\footnotetext{
36- Notons la résistance de la traction hippomobile par rapport à la traction motorisée. MARCHI, 2007, p. 17-18 faisant référence à LAMMING, 2003, qui a remarqué la persistance de la traction hippomobile sur certains réseaux miniers en Grande-Bretagne jusque dans les années 1880 .

37- DEMONFAUCON, 2003, carte postale, p. 11.

38- La loi Migneret (1865) a cependant déjà fourni un premier cadre juridique aux réseaux d'intérêt local. Voir WOLKOWITSCH, 2004, p. 30-38, et WOLKOWITSCH, 2001, p. 410-433 (textes complets des lois).
} 
l'heure, les locomotives sont chauffées, pour éviter les fumées, au coke de gaz ${ }^{39}$ fourni par l'usine à gaz de Royan. Et les journalistes de L'Abeille de Seine-etOise de relever : "le service se faisait dans des conditions de propreté souvent supérieures à celles que procure la marche des moteurs Diesel plus ou moins bien réglés ${ }^{40}$. " Le tramway remporte d'emblée un tel succès qu'il faut rapidement acheter de nouvelles locomotives.

Il s'agit d'un réseau que l'on peut qualifier de "touristico-urbain ", dans la mesure où il répond à la fois aux besoins de la population locale (il fonctionne toute l'année, cependant durant la saison hivernale les circulations sont réduites) mais aussi (et surtout) aux désirs des touristes qui peuvent ainsi commodément passer d'une conche à l'autre. En 1897, suite au prolongement jusqu'à la Grande Côte, une correspondance est établie avec le tramway forestier de la Coubre.

En 1923, la Société générale des tramways de Royan ${ }^{41}$ reprend la concession du tramway forestier de la Coubre et le reconstruit en voie de $60 \mathrm{~cm}$. C'est donc la conversion aux standards Decauville d'une exploitation ferroviaire dont la conception n'en relevait pas initialement. Désormais, il n'y a plus qu'un seul réseau au caractère touristique de plus en plus marqué. La Société générale des tramways de Royan, sous la direction de $M$. Nougarède, l'exploite activement dès l'année suivante avec des automotrices à essence ${ }^{42}$. Elle réorganise le parcours sous forme d'excursion : ainsi, les personnels du tramway guident les voyageurs sur les plages ${ }^{43}$. Elle fait aussi éditer des carnets de cartes postales vendus à bord des trains par les contrôleurs-receveurs ${ }^{44}$. Ce pari sur le tourisme permet aux Tramways de Royan de perdurer au-delà des autres 'petits trains des plages'. Ils succombent toutefois à la suite des dégâts causés par les violents bombardements dont Royan est la victime à la fin du second conflit mondial.

Archétypes du petit train Decauville, les Tramways de Royan laissent un souvenir impérissable aux 'ferrovipathes'. Ils inspirent à partir des années

39- Ce n'est pas tout à fait une première. Dans le même but, on a eu recours au coke de gaz pour les locomotives de l'Exposition Universelle de 1889. Le coke de gaz est issu de la distillation de la houille pour la fourniture de gaz d'éclairage.

40- BAILLY, 1989, p. 80.

41- Il s'agit du second concessionnaire des Tramways de Royan, le premier concessionnaire étant naturellement les Établissements Decauville.

42- DEMONFAUCON, 2003, p. 81.

43- Ibidem, p. 83.

44- Ibid., commentaire p. 82. Des échantillons de cartes postales figurent p. 82-85. 
1950 la création de nouveaux chemins de fer touristiques : notamment les tout premiers d'entre eux, le Tramway forestier du Cap Ferret et le Tramway touristique de Saint-Trojan ${ }^{45}$.

\section{Le tramway du parc de Royan}

Nous disposons de peu d'informations sur le tramway du parc de Royan ; nous ne connaissons la date ni de son établissement, ni de la fin de son exploitation. Mais, à l'évidence, il ne s'agit d'aucun des deux premiers réseaux déjà mentionnés, et encore moins du quatrième que nous évoquerons. Le tramway du parc de Royan est mentionné succinctement dans le guide de Victor Billaud : "un tramway semblable à celui du Jardin d'Acclimatation [...] dessert la forêt en suivant les grandes avenues intérieures et le boulevard de la $\mathrm{mer}^{46}$. " Cette référence nous laisse penser que le tramway utilise probablement le système Decauville. Une carte jointe au guide fait apparaître un itinéraire tortueux épousant le tracé des rues.

\section{Le " tramway des enfants"}

Le " tramway des enfants " est une courte ligne (600 mètres) à voie de $60 \mathrm{~cm}$ installée dans un parc privé, le "Stade de l'Oasis». Il connaît une existence éphémère : aménagé vers 1923, il aurait disparu autour de 1929. Son matériel ? " De minuscules voitures à 4 places et 2 locomotives inesthétiques, à moteur à essence! L'une de forme vapeur et l'autre imitant grossièrement une BB du P.O. ${ }^{47}$. " Ecartement mis à part, il n'a rien à voir avec les conceptions Decauville.

\section{Un modèle Decauville}

Les différents tramways évoqués jusque-là ne sont pas tous liés aux conceptions Decauville. Mais Paul Decauville a su, le premier, ériger les petits trains en un véritable système.

Tout d'abord, les petits trains Decauville constituent indéniablement une innovation. Paul Decauville est l'inventeur d'un matériel ferroviaire dont

\footnotetext{
45- Jacques Milet caresse le rêve de faire revivre les Tramways de Royan avant de créer le Tramway forestier du Cap Ferret en 1952. Le médecin militaire Pol Gala, avec des membres de sa famille et des amis passionnés de trains, essaie de ressusciter les mêmes tramways sans y parvenir. Il se rabat alors sur l'île d'Oléron voisine, où le Tramway touristique de Saint-Trojan est inauguré en 1963.

46- Voir note 24.

47- VERGER et CHAPUIS, 1977, p. 8. Une photo, p. 7, révèle un matériel d'allure peu ferroviaire et donc probablement de conception artisanale.
} 
la légèreté et la simplicité d'utilisation le désignent comme le support idéal pour des chemins de fer de dimension modeste, construits rapidement et sans frais excessifs. Ces petits trains peuvent ainsi répondre aux besoins d'opérateurs qui, généralement, ne sont pas des professionnels du transport ferroviaire (promoteurs immobiliers, architectes, hôteliers, restaurateurs, entrepreneurs de travaux publics ou de transport non ferroviaire $)^{48}$ et qui n'auraient pas eu accès aux 'grands' trains.

Pourtant, malgré leur petit format, les trains Decauville sont de vrais chemins de fer, modernes pour l'époque qui plus est. Avec des trains qui auraient pu passer pour des "sous-produits ferroviaires ", Paul Decauville a réussi non seulement à faire des produits attractifs, mais aussi à lancer un phénomène de mode.

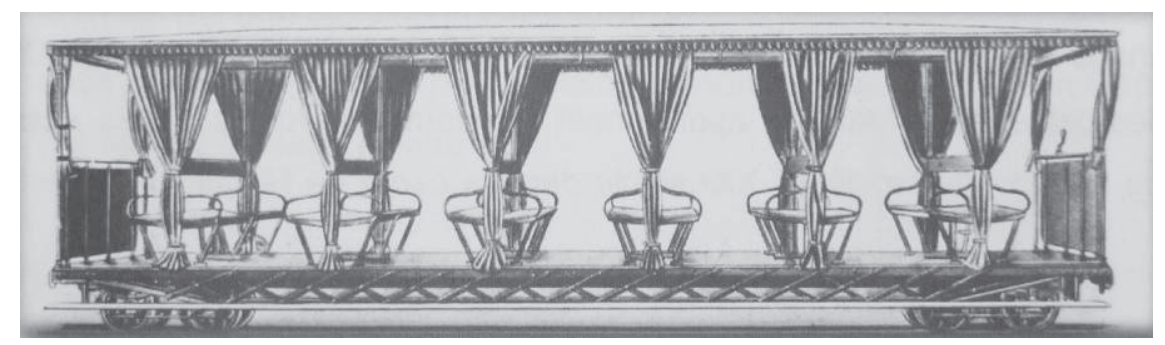

Figure 4. Baladeuse construite par les Établissements Decauville, vers 1890, pour les Tramways de Royan, Mairie de Saint-Trojan, dossier "STTST - Petit train ", section "Petit tramway touristique ", copie d'une gravure, 21 x 29,7 cm. Figure reproduite dans Stéphane Calonnec, "Dynamique rurale, environnementalisme et tourisme de masse dans le sud de l'île d'Oléron : la Société du tramway touristique de Saint-Trojan, 1959-2008 ", mémoire de Master 1 (histoire), université de La Rochelle, sous la dir. de Guy Martinière, 2008, Annexe, figure 17, p. XXVIII.

Car le 'Decauville' s'identifie du premier coup d'œil par ses matériels roulants originaux qui participent de sa "ludicité ». Le format miniature des locomotives et voitures, des gares et installations diverses possède un charme particulier comme l'ont fait remarquer les journalistes de l'époque. Les petits trains se caractérisent, presque depuis les origines, par l'emploi quasiment général de baladeuses, créations des Établissements Decauville ${ }^{49}$. Ces voitures

48- Paul Decauville lui-même n'est pas, au départ, « quelqu'un du métier » : c'est un fermier industrieux, archétype de l'entrepreneur du XIX ${ }^{e}$ siècle. Voir supra.

49- Elles seraient inspirées du premier étage des voitures à impériale des chemins de fer de banlieue. 
ouvertes sur les côtés permettent une promenade 'le nez au vent' et un contact direct avec la nature environnante (odeurs des forêts de pins, par exemple). Les faibles vitesses pratiquées rendent l'expérience agréable. L'aspect général des baladeuses a peu changé en plus d'un siècle : entre les baladeuses d'hier et d'aujourd'hui seuls ont disparu les longs rideaux rayés qu'affectionnaient apparemment nos aînés (fig. 4 et 5). La pérennité du concept, pendant plus d'un siècle, traduit selon nous sa remarquable adaptation au tourisme ferroviaire ${ }^{50}$.

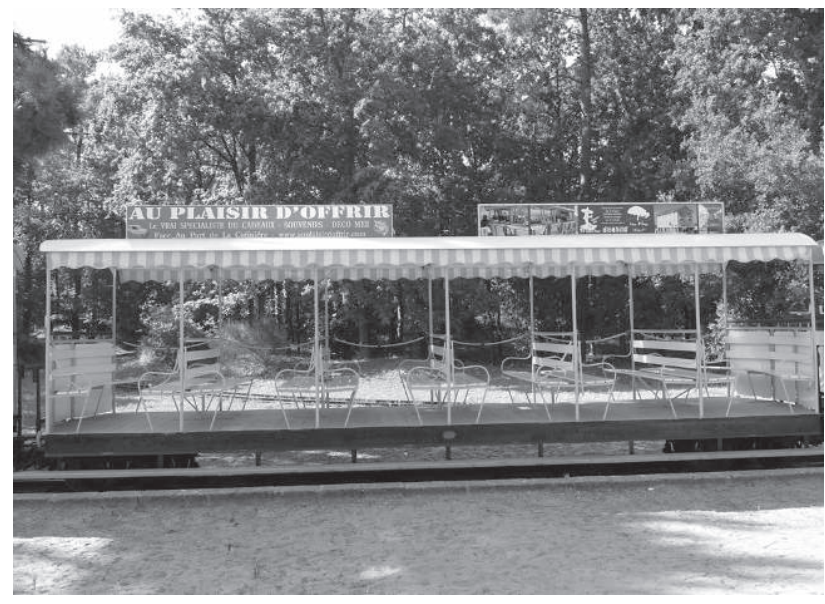

Figure 5. Baladeuse employée de nos jours sur le P'tit Train de Saint-Trojan. II s'agit d'une réplique du modèle précédent. Cl. J.-J. Marchi.

Grâce aux petits trains, Paul Decauville a développé les pratiques touristiques et de loisirs. Ses réalisations, notamment le petit train du Jardin d'Acclimatation et le petit train de l'Exposition Universelle de 1889, ont eu un tel retentissement qu'elles ont popularisé l'idée de la balade en petit train, une forme de tourisme ferroviaire.

L'histoire aurait pu s'arrêter là. Or, le matériel Decauville renaît dans la seconde moitié du $\mathrm{xx}^{\mathrm{e}}$ siècle : d'un côté, la multiplication des petits trains ludiques et leur diffusion aux parcs d'attractions traduit une adaptation des concepts Decauville (fig. 6) ; de l'autre, le réemploi de matériels Decauville anciens par des « ferrovipathes", créateurs de chemins de fer touristiques, met

50- Les préférences du public en termes de matériels roulants traduisent bien cette adaptation. Ainsi, sur les Tramways de Royan, les voitures fermées ont peu circulé, boudées par les voyageurs qui leur préféraient les baladeuses, et les premières ont été retirées de la circulation prématurément, finissant leur carrière comme abris de jardin. Voir DEMONFAUCON, 2003, p. 35 et 93. De nos jours, certains chemins de fer touristiques à voie normale ont construit des voitures d'un aspect proche des baladeuses, et qui rencontrent un large succès. 
en évidence la pérennité de matériels désormais valorisés en tant que patrimoine ferroviaire.

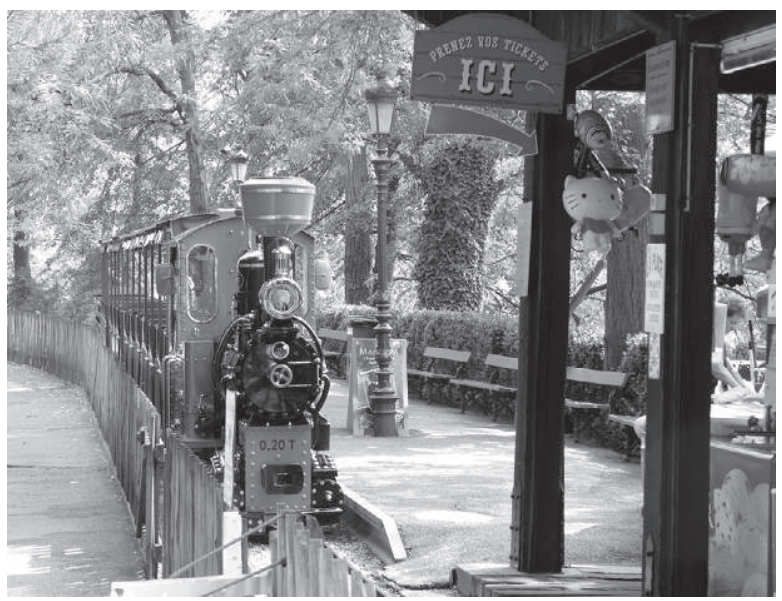

Figure 6. Le petit train du Parc de la Tête d'Or à Lyon : le chemin de fer ludique et son folklore. Cl. J.-J. Marchi.

Le petit train ludique reste de nos jours peu éloigné des concepts Decauville. Comme jadis, il est employé pour mettre un peu d'animation près des plages ou dans les jardins publics. En outre, avec le développement des pratiques de loisirs, à l'origine d'offres nouvelles, il s'est diffusé dans les bases de loisirs et les parcs d'attractions : nous trouvons des petits trains à Disneyland Paris ${ }^{51}$, à la Mer de Sable (Oise) ${ }^{52}$, au Pal (Allier), au Domaine Paul Ricard de Méjanes (Bouches-du-Rhône) pour ne citer que quelques exemples. Ils constituent en effet des auxiliaires précieux à la fois pour divertir les visiteurs et les faire circuler commodément et rapidement à l'intérieur de la zone d'animation ${ }^{53}$.

Lémergence d'une sensibilité pour le patrimoine ferroviaire se traduit par la nostalgie des petits trains d'autrefois ${ }^{54}$. Le matériel Decauville encore valide revit dans les mains des 'ferrovipathes'. En effet, les vestiges du système Decauville permettent le démarrage ou redémarrage rapide et économique d'exploitations touristiques à voie étroite par l'utilisation de rails légers, faciles

51- Walt Disney était un «ferrovipathe ». Il s'était construit chez lui, pour le plaisir, un petit train à l'échelle $1 / 8$. Les petits trains ont toujours été partie intégrante de ses parcs d'attraction. Voir WHITWORTH, 1996, p. 109-110.

52- Il s'agit du premier parc d'attraction à thème français, inauguré par Jean Richard en 1963. 53- SCHOOLFIELD, 2006, et WHITWORTH, 1996. Ce sont les gérants de parcs d'attraction qui ont donné l'opportunité aux « ferrovipathes » André Ravery et Patrick Mourot de faire leurs premières armes. Voir MARCHI, 2010, p. 364-365, p. 366-368.

54- Voir note 45. Nous avons déjà évoqué le rôle joué par la nostalgie des Tramways de Royan. 
à mettre en place une fois récupérés dans des sucreries, des carrières, des mines abandonnées ${ }^{55}$. Par ailleurs, les matériels roulants à divers écartements, fabriqués par les Établissements Decauville depuis les origines jusqu'en 19681969, sont également réutilisés avec profit ${ }^{56}$. Certains de ces matériels sont encore employés de nos jours à la fois par des chemins de fer touristiques (les locomotives à vapeur Decauville sont particulièrement bien représentées en voie de 60) et pour l'organisation de trains spéciaux (par exemple l'autorail X2403 des Chemins de fer de Haute-Auvergne en voie normale est une fabrication Decauville) ${ }^{57}$.

À deux époques, sur plus d'un siècle, Paul Decauville, ses conceptions et ses productions, participent activement (mais pas exclusivement) à l'essor du tourisme ferroviaire. Innovant et bien 'marketé' par son créateur, le 'Decauville' représente à la fin du xix ${ }^{e}$ siècle le modèle le plus abouti de petit train ludique. Dans la seconde moitié du Xx $x^{\mathrm{e}}$ siècle, survivant à son créateur, le 'Decauville' reste l'archétype du petit train, tant auprès des exploitants de parcs d'attractions et de bases de loisirs qu'auprès de 'ferrovipathes' nostalgiques. Nous pouvons donc parler d'un véritable 'modèle Decauville'.

55- Cas, entre autres, du Tramway Pithiviers - Toury (TPT) dont une section est reprise par l'Association du Musée des transports de Pithiviers (Loiret), du Chemin de fer touristique du Tarn (CFTT), du « Tacot des lacs » (Seine-et-Marne).

56- En 1956, le petit train d'André Ravery circulant au «Joy Parc » de Meaux est tiré par une locomotive à vapeur Decauville (voir note 53). Une locomotive à vapeur Decauville est aussi le premier matériel de traction du Chemin de fer touristique de Meyzieu, inauguré en 1962 près de Lyon.

57- Signalons aussi des fabrications neuves pour des trains touristiques de Haute-Savoie : le Tramway du Mont-Blanc, le Chemin de fer du Montenvers - Mer de Glace, le « Mont-Blanc Express ». 


\section{Bibliographie}

ACOVA, 2002 : ACOVA (Association pour la conservation occitane de véhicules anciens), Le Chemin de fer touristique du Tarn, plaquette de présentation, Toulouse, ACOVA, édition 2002.

ARRIVETZ, 2002 : Arrivetz, Jean, "Histoire de la Société des Chemins de Fer Touristiques et de Montagne (CFTM) ", Chemins de fer régionaux et urbains, $\mathrm{n}^{\circ} 289$ (2002/1), p. 13-18.

AUPHAN, 1999 : Auphan, Etienne, "Les chemins de fer touristiques entre patrimoine et tourisme récréatif ", in "Le Patrimoine ferroviaire : enjeux, bilans et perspectives ", Actes du sixième colloque de l'AHICF (Mulhouse, 23-26 septembre 1998), en collaboration avec le CILAC, Comité d'information et de liaison pour l'archéologie, l'étude et la mise en valeur du patrimoine industriel, et le Musée français du Chemin de fer, Revue d'histoire des chemins de fer, $\mathrm{n}^{\circ}$ 20-21 (printemps-automne), 1999, p. 255-268.

BAILLY, 1989 : Bailly, Roger, Decauville, ce nom qui fit le tour du monde, La Mée-sur-Seine, Amatteis, 1989.

BEIGBEDER et PRADAYROL, 1979 : Beigbeder, G. et Pradayrol, Jacques, "Le chemin de fer du Festiniog ", Voie étroite, n 50 (1979/1), p. 10-31.

BILLAUD, 1890 : Billaud, Victor, Royan et ses environs, guide du touriste, Royan, Victor Billaud Éditeur, 1890.

BOUCHEZ, 1964 : Bouchez, Pierre, "Le chemin de fer Decauville à l'exposition de 1889 ", Chemins de fer secondaires, n 64 (1964/4), p. 3-10.

CALONNEC, 2008 : Calonnec, Stéphane, «Dynamique rurale, environnementalisme et tourisme de masse dans le sud de l'île d'Oléron : la Société du tramway touristique de Saint-Trojan, 1959-2008 ", mémoire de Master 1 (histoire), université de La Rochelle, sous la dir. de Guy Martinière, 2008, t. 1, texte, 95 p. ; t. 2, annexes, 30 p.

CHANUC et DURBAIN, 2005 : Chanuc, Lucien et Durbain, Patrice, Les Trains du Médoc, Breil-sur-Roya, Les Éditions du Cabri, 2005.

CORBIN, 1988 : Corbin, Alain, Le Territoire du vide : l'Occident et le désir de rivage (1750-1840), Paris, Aubier, 1988.

DEMONFAUCON, 2003 : Demonfaucon, Cédric, Les Tramways de Royan à Ronce-les-Bains, Saint-Cyr-sur-Loire, Alan Suton, coll. " Mémoire en images ", 2003.

DOLLFUS et GEOFFROY, 1938 : Dollfus, Charles et Geoffroy (de), Edgar, Histoire de la locomotion terrestre. Les chemins de fer, Paris, L'Illustration, $2^{\mathrm{e}}$ éd., 1938. 
DUPUYOO, 2007 : Dupuyoo, Luc, Les Petits Trains du Cap-Ferret, Bordeaux, Éditions Confluences, 2007.

GEIGER, 1970 : Geiger, Maurice, « Les chemins de fer touristiques et d'attraction ", Chemins de fer régionaux et urbains, $\mathrm{n}^{\circ} 100$ (1970/4), p. 77-84.

GIUNTINI, 2003 : Giuntini, Andrea, " Le tourisme ferroviaire en Italie des origines jusqu'à la création des 'trains populaires' ", in Laurent Tissot (sous la dir.), Construction d'une industrie touristique aux $19^{\circ}$ et $20^{\circ}$ siècles. Perspectives internationales, Neuchâtel, Alphil, coll. " Histoire ", 2003, p. 59-81.

LAEDERICH, 1986 : Laederich, Pierre, "Les tramways du Cap-Ferret ", Connaissance du rail, n ${ }^{\circ} 69$ (juillet-août 1986), p. 4-14.

LAMMING, 2003 : Lamming, Clive, "Retour aux origines et aux années 1820 : de l'atelier de charronnage primitif anglais aux premiers dépôts organisés en France par Marc Seguin ", in Christian Chevandier et Philippe Mioche (sous la dir.), "Ateliers et dépôts du Matériel ferroviaire, deux siècles d'histoire ", Actes du dixième colloque de l'AHICF (Arles, avril 2002), Revue d'histoire des chemins de fer, $\mathrm{n}^{\circ} 28-29$ (printemps-automne 2003), p. 257-274.

MARCHI, 2007 : Marchi, Jean-Jacques, "Les chemins de fer touristiques entre nostalgie et innovation (1957-2007) ", mémoire de Master èssciences économiques, option histoire économique, sous la direction du pr. B. Blancheton, université Montesquieu Bordeaux IV, 2007.

MARCHI, 2008 : Marchi, Jean-Jacques, "Les chemins de fer touristiques : des 'petits trains' singuliers et pluriels", Revue d'histoire des chemins de fer, $\mathrm{n}^{\circ} 38$ (printemps 2008), p. 213-235.

MARCHI, 2010 : Marchi, Jean-Jacques, "Le tourisme ferroviaire à petite vitesse ", thèse de doctorat ès-sciences économiques, sous la direction du pr. B. Blancheton, université Montesquieu Bordeaux IV, 2010.

SCHOOLFIELD, 2006 : Schoolfield, Jeremy, «Steady like a train », Funworld, The official magazine of the international association of amusement parks and attractions, volume XXII, n 7 (juillet 2006), p. 60-62.

THEVENIN, 1994 : Thevenin, Jacques, "Les tramways du Cap Ferret ", Chemins de fer régionaux et urbains, $\mathrm{n}^{\circ} 244$ (1994/4), p. 3-17.

VERGER et CHAPUIS, 1977 : Verger, R., et Chapuis, Jacques, « Les tramways de Royan ", Chemins de fer régionaux et urbains, no 139 (1977/1), p. 2-87.

WHITWORTH, 1996 : Whitworth, A.W., "Theme Parks Trains That Could", Funworld, The official magazine of the international association of amusement parks and attractions, volume XII, $\mathrm{n}^{\circ} 11$ (nov. 1996), p. 105-115. 
WOLKOWITSCH, 2001 : Wolkowitsch, Maurice (sous la dir.), «Le chemin de fer à la conquête des campagnes, l'aménagement du territoire par les réseaux dits 'secondaires' en France, histoire et patrimoine, 1865-2001 ", Actes du $9^{\mathrm{e}}$ colloque de l'AHICF (Châteauroux, sept. 2011), Revue d'histoire des chemins de fer, 24-25 (2001).

WOLKOWITSCH, 2004 : Wolkowitsch, Maurice, "le siècle des chemins de fer secondaires en France, 1865-1963. Les entreprises, les réseaux, le trafic ", Revue d'histoire des chemins de fer, 30 (printemps 2004). 
